\title{
Activity of fibroblast growth factor receptor inhibitors TKI258, ponatinib and AZD4547 against TPR-FGFR1 fusion
}

\author{
XU-HUA QIU* , FENG LI* , HONG-QIN CAO, JING-JING SHAO, \\ JIAN-GANG MEI, HAN-QING LI and YONG-PING ZHAI \\ Department of Hematology, Jinling Hospital, Nanjing, Jiangsu 210002, P.R. China \\ Received November 19, 2015; Accepted November 29, 2016
}

DOI: $10.3892 / \mathrm{mmr} .2017 .6140$

\begin{abstract}
EMS) is a rare disease characterized by the constitutive activation of fibroblast growth factor receptor 1 (FGFR1). To date, four cases of EMS with the chromosomal translocation, $\mathrm{t}(1 ; 8)(\mathrm{q} 25 ; \mathrm{p} 11.2)$, have been reported. In the present study, TPR-FGFR1-expressing Baf3 cells were established and confirmed by polymerase chain reaction. To identify the most promising drug for EMS, the activities and associated mechanism of three tyrosine kinase inhibitors (TKIs), TKI258, ponatinib and AZD4547, against TPR-FGFR1 were tested by MTT assay, flow cytometry and western blot. The data demonstrated that TPR-FGFR1 was localized in the cytoplasm, and was able to transform interleukin-3-dependent hematopoietic Baf3 cells into growth factor-independent cells. All of the three TKIs markedly inhibited the proliferation of TPR-FGFR1-expressing Baf3 cells, and the activation of FGFR1 and the downstream signaling molecules, extracellular signal-regulated kinase 1/2, phospholipiase $C \gamma$ and signal transducer and activator of transcription 5. AZD4547 was the most efficient drug, and TKI258 was the least. By contrast, no significant difference was found among the three drugs on their effect on cell apoptosis. Taken together, the data obtained in the present study suggested that AZD4547 had increased potency, compared with TKI258 and ponatinib, for the treatment of EMS.
\end{abstract}

\section{Introduction}

8p11 myeloproliferative syndrome (EMS) is a rare, aggressive disease, which progresses to acute leukemia within 1-2 years

Correspondence to: Dr Yong-Ping Zhai, Department of Hematology, Jinling Hospital, 305 East Zhongshan Road, Nanjing, Jiangsu 210002, P.R. China

E-mail: yongping_zhai@163.com

*Contributed equally

Key words: 8p11 myeloproliferative syndrome, fibroblast growth factor receptor 1, tyrosine kinase inhibitor, TKI258, ponatinib, AZD4547 following diagnosis (1). It is caused by the constitutive activation of the fibroblast growth factor receptor 1 (FGFR1) gene by fusion to various partner genes. To date, 15 FGFR1 partner genes have been identified, including $B C R$ (22q11), NUP98 (11p15), HERV-K (19q13), MYO18A (17q23), ZNF198 (13q12), CPSF6 (12q15), FGFR1OP2 (12p11), CEP110 (9q33), TIFI (7q34), CUX1 (7q22), FOP (6q27), LRRFIP1 (2q37), TPR (1q25) (2), RANBP2/NUP358 (2q12) (3) and SQSTM1 (5q35) (4).

The TPR-FGFR1 arrangement was first identified in our previous study (2) in a patient, who presented with myeloproliferative neoplasm-like symptoms. In this case, exon 23 of the TPR gene was fused to exon 13 of FGFR1. Another case of TPR-FGFRl rearrangement, with a novel breakpoint of exon 22 of the TPR gene, has also been reported (5). However, the biological function of this fusion remains to be elucidated.

The constitutive activation of FGFR1 kinase is the primary cause of EMS, which suggests the potential of FGFR1 kinase as a promising target for the treatment of EMS. Previous studies have shown that SU5402, PD173074 and PKC412 potently inhibit the proliferation of ZNF198-FGFR1-transformed Baf3 cells (6). However, the results did not translate into clinical benefits, and novel FGFR1-targeted compounds are required for EMS therapy (7).

TKI258, ponatinib and AZD4547 are three clinically evaluated TKIs, which target FGFR1 with enzyme half maximal inhibitory concentration $\left(\mathrm{IC}_{50}\right)$ values $<10 \mathrm{nmol} / \mathrm{l}(6)$. Previous studies have also indicated that TKI258, ponatinib and AZD4547 are active against certain EMS-associated fusion genes (8-11).

In the present study, the transforming activity of TPR-FGFR1 was analyzed, and the effects of TKI258, ponatinib, and AZD4547 on the fusion gene were compared in vitro to identify the drug with the most potential for the treatment of EMS.

\section{Materials and methods}

Constructs. The TPR-FGFR1/pEZ-M03 plasmid was generated using Gateway cloning technology. Briefly, TPR1-23 was amplified from the CCS-Z8318-M03 plasmid (FulenGen Co., Ltd., Guangzhou, China) using the following primers: Forward 5'-GGAAGTTCGAACCATGGCGGCGGTGTT GCAGCAAGT-3' and reverse 5'-CACTGGAGTCAGCA 
GACACCTGTTGTTCCATGCTCTCTATGGC-3'. DNA was first denatured for $10 \mathrm{~min}$ at $94^{\circ} \mathrm{C}$, and then amplified using 25 cycles of $30 \mathrm{sec}$ at $94^{\circ} \mathrm{C}, 30 \mathrm{sec}$ at $58^{\circ} \mathrm{C}$ and 2 min at $72^{\circ} \mathrm{C}$. The $F G F R 1$ moiety was liberated from the EX-Z0528-M03 plasmid (FulenGen Co., Ltd.) using the following primers: Forward 5'-GCCATAGAGAGCATG GAACAACAGGTGTCT GCT GACTCCAGT G-3' and reverse 5'-TGCGGCCGCACTCGAGGTAGCGGCGTTTGA GTCCGCCATTGG-3'. The TPR and FGFRI fragments were ligated into the pDONR ${ }^{\mathrm{TM}}$ vector (FulenGen Co., Ltd.) using a Fast-Fusion $^{\mathrm{TM}}$ kit (FulenGen Co., Ltd.). The fusion gene was then transferred into pEZ-M03/GFP via the Gateway LR cloning reaction (EZShuttle ${ }^{\mathrm{TM}}$ LR Recombination Cloning System, Genecopoeia, Rockville, MD, USA).

The full-length TPR-FGFRI was amplified from the TPR-FGFR1/pEZ-M03 plasmid using the following primers: Forward 5'-CTTAGA ATTCGCCACCATGGCGGCGGT GTT GCAGCA AGT CCT GGA GCGCACGGA-3' and reverse 5'-CTTACTCGAGCTAGCGGCGTTTGAGTCCGC CATTGGCAAGCTGGGCTGGGTG-3'. The target DNA was amplified using 25 cycles of $30 \mathrm{sec}$ at $94^{\circ} \mathrm{C}, 30 \mathrm{sec}$ at $58^{\circ} \mathrm{C}$ and $2.5 \mathrm{~min}$ at $72^{\circ} \mathrm{C}$. This was then cloned into the EcoRI/XhoI site of the pMSCV (ClontechLaboratories, Inc., Mountainview, CA, USA).

The successful construction of TPR-FGFR1/pEZ-M03 and TPR-FGFR1/pMSCV were validated using gene sequencing.

Cell lines. All cell lines were obtained from the China Infrastructure of Cell Line Resources (Beijing, China). Baf3 cells were cultured in RPMI 1640 supplemented with $10 \%$ fetal calf serum containing interleukin (IL) $3(10 \mathrm{ng} / \mathrm{ml}$; eBioscience, Inc., Vienna, Austria). COS-1 and 293T cells were cultured in Dulbecco's modified Eagle's medium containing 10\% newborn calf serum (Zhejiang Evergreen Biologicals, Zhejiang, China).

Subcellular localization of TPR-FGFR1. COS-1 cells $\left(1 \times 10^{5} / \mathrm{ml}\right)$ were cultured $\left(5 \% \mathrm{CO}_{2}, 37^{\circ} \mathrm{C}\right)$ in a six-well microplate with a polylysine-coated coverslip. After $24 \mathrm{~h}$, the cells were transfected with TPR-FGFR1/pEZ-M03 using Thermo Scientific TurboFect transfection reagent (Thermo Fisher Scientific, Inc., Rockford, IL, USA) according to the manufacturer's protocol. Subsequently, the protein localization (green fluorescence) was detected using fluorescence microscopy.

Establishment of TPR-FGFR1-expressing Baf 3 cells. Retroviral stock was generated by co-transfection of 293T cells with TPR-FGFR1/pMSCV or empty vector and two packaging constructs (VSVG and MD2) provided by Professor Quan Zhao (Nanjing University, Nanjing, China). At 48 h post-transfection, the supernatant was collected and stored at $-70^{\circ} \mathrm{C}$. For the transfection, the virus-containing supernatant was added to the Baf3 cells $\left(5 \times 10^{5} / \mathrm{ml}\right)$ together with $10 \mathrm{mg} / \mathrm{ml}$ polybrene, and cultured at $37^{\circ} \mathrm{C}$ for $72 \mathrm{~h}$. The cells were then selected by the addition of $1 \mathrm{mg} / \mathrm{ml} \mathrm{G} 418$. The medium was replaced every 4 days. After 5 weeks, the resistant cells were collected for use in the subsequent experiments.

Fusion gene characterization. RNA was extracted from cells using TRIzol reagent (Invitrogen; Thermo Fisher Scientific,
Inc.), and $1 \mu \mathrm{g}$ RNA was reverse transcribed into cDNA with PrimeScript $^{\mathrm{TM}}$ RT Master Mix (Takara Bio, Inc., Otsu, Japan) according to the manufacturer's protocol. Polymerase chain reaction (PCR) analysis was performed using a BIO-RAD T100 Thermal Cycler (Bio-Rad Laboratories, Inc., Hercules, CA, USA). The primer combinations used to detect the fusion gene were as follows: Sense, 5'-GCCACATTGAAACAGCAC CTC-3' and antisense 5'-AGCCGTGATGGCCGAAC-3'. The target DNA was amplified with Takara PCR Amplification Kit (Takara Bio, Inc.) using 30 cycles of $30 \mathrm{sec}$ at $94^{\circ} \mathrm{C}, 30 \mathrm{sec}$ at $60^{\circ} \mathrm{C}$, and $40 \mathrm{sec}$ at $72^{\circ} \mathrm{C}$.

Proliferation assays. The cells were seeded into a 96-well plate at $1 \times 10^{4} / \mathrm{ml}(100 \mu \mathrm{l} /$ well $)$ with or without IL3 $(10 \mathrm{ng} / \mathrm{ml})$ at $37^{\circ} \mathrm{C}$. The growth curve was constructed by counting the number of viable cells, which excluded trypan blue, each day with microscope (Olympus CX41). The selected cells were then seeded into a 96 -well plate at $1 \times 10^{5} / \mathrm{ml}(100 \mu \mathrm{l} /$ well $)$ and treated with different concentrations of the TKIs (Selleck Chemicals, Shanghai, China), TKI258, ponatinib and AZD4547, ranging between 1 and $1,000 \mathrm{nmol} / 1$ with half-log increments at $37^{\circ} \mathrm{C}$. Cells treated with DMSO were used as a control. After $48 \mathrm{~h}, 20 \mathrm{ml}$ of $5 \mathrm{mg} / \mathrm{ml}$ methylthiazolyldiphenyl-tetrazolium bromide was added to each well, and cultured at $37^{\circ} \mathrm{C}$ for $4 \mathrm{~h}$. The supernatant was removed carefully following centrifugation at $1,600 \times \mathrm{g}$, room temperature, for $30 \mathrm{~min}$. Subsequently, $100 \mu 1$ DMSO was added and absorbance was detected at $490 \mathrm{~nm}$ (Bio-Rad iMark Microplate Reader; Bio-Rad Laboratories, Inc.).

Apoptosis assay. Cells were treated with the different concentrations of the TKIs for $24 \mathrm{~h}$. Subsequently, apoptosis was detected using an Annexin V/PI apoptosis kit (7Sea Biotechnology, Shanghai, China) according to the manufacturer's protocol. The stained cells were then detected using the FACS Calibur system (Miltenyi Biotec GmbH, Cologne, Germany).

Western blot analysis. The cells were serum starved overnight and treated with the different concentrations of the TKIs for $90 \mathrm{~min}$. The cells were lysed on ice with RIPA cell lysis buffer containing $1 \mathrm{mmol} / \mathrm{l} \mathrm{PMSF}$ (Beyotime Institute of Biotechnology, Shanghai, China). The lysed cells were harvested, transferred into new tubes, and centrifuged for $10 \mathrm{~min}$ at $12,000 \times \mathrm{g}$ at $4^{\circ} \mathrm{C}$, following which the supernatants were collected. The protein concentrations were determined using a BCA kit (Beijing Solarbio Science \& Technology Co., Ltd., Beijing, China). A total of $30 \mathrm{mg}$ protein per group was electrophoresed on a $30 \%$ sodium dodecyl sulfate-polyacrylamide electrophoresis gel and transferred onto a PVDF membrane. Following three washes with TBST, the membrane was blocked by 5\% BSA (Sigma-Aldrich; Merck Millipore, Darmstadt, Germany) for $2 \mathrm{~h}$ at room temperature. The primary antibodies, rabbit anti-FGFR1 (cat. no. sc-121; 1:100; Santa Cruz Biotechnology Inc., Dallas, TX, USA), rabbit anti-phospho-FGFR1 (cat. no. 3461; 1:1,000; Cell Signaling Technology, Inc. Beverly, MA, USA), rabbit anti-phospho-signaling transducer and activator of transcription 5 (STAT5; cat. no. 4322; 1:1,000; Cell Signaling Technology, Inc.), rabbit anti-phospho-phospholipase $\gamma$ 
(PLC $\gamma$; cat. no. 8713; 1:1,000; Cell Signaling Technology, Inc.), rabbit anti-phospho-extracellular signal-regulated kinase (ERK; cat. no. 4695; 1:1,000; Cell Signaling Technology, Inc.), rabbit anti-phospho-Akt (cat. no. 2965; 1:1,000; Cell Signaling Technology, Inc.) and rabbit anti- $\beta$-actin (cat. no. AA128; 1:1,000; Beyotime Institute of Biotechnology), were used for the incubation overnight at $4^{\circ} \mathrm{C}$. Following this, the membrane was washed with TBST for $10 \mathrm{~min}$ at room temperature three times. Following $2 \mathrm{~h}$ of incubation with horseradish peroxidase-conjugated anti-rabbit IgG antibody (cat. no. A0208, 1:1,000, Beyotime Institute of Biotechnology) at room temperature, the membrane was washed three times with TBST and detected with enhanced chemiluminescence (GE Healthcare Life Sciences, Little Chalfont, UK).

Statistical analysis. The results are expressed as the mean \pm standard error of the mean. Student's t-test was used to compare data with SPSS software version 19.0 (IBM SPSS, Armonk, NY, USA). $\mathrm{P}<0.05$ was considered to indicate a statistically significant difference.

\section{Results}

TPR-FGFR1 is localized in the cytoplasm. In order to investigate the subcellular localization of the fusion gene, COS-1 cells were transiently transfected with TPR-FGFR1/pEZ-M03. GFP was conjugated to the carboxyl terminus of the fusion gene. As shown in Fig. 1, TPR-FGFR1 was exclusively localized in the cytoplasm.

TPR-FGFRl is expressed in TPR-FGFRl-transfected Baf3 cells. The selected TPR-FGFR1-transfected Baf3 cells were analyzed for the expression of the fusion gene using reverse transcription (RT)-PCR analysis. Empty vector (pMSCVneo)-transfected cells were used as a negative control. The PCR product of the TRP-FGFR1/pMSCV construct, which was confirmed using gene sequencing, was also electrophoresed as a positive control. Data showed that TPR-FGFR1 was expressed in the selected Baf3 cells (Fig. 2). These cells were referred to as TPR-FGFR1-expressing Baf3 cells in the subsequent experiment.

TPR-FGFR1 transforms Baf3 cells into IL3-independent cells. The growth dependence of IL3 was evaluated to investigate the transforming activity of TPR-FGFR1. As shown in Fig. 3, all the cells transfected with the empty vector (pMSCVneo) died within 2 days, whereas the TPR-FGFR1-expressing Baf3 cells were found to grow in an IL3-independent manner. The growth rate of theTPR-FGFR1-expressing Baf3 cells was marginally lower, compared with that in the pMSCV-neo transfected cells with IL3.

TKI258, ponatinib and AZD4547 inhibit the proliferation of TPR-FGFR1-expressing Baf3 cells. Previous studies have investigated the activities of TKI258, ponatinib and AZD4547 against ZNF198-FGFR1, BCR-FGFR1, CUX1-FGFR1 and FGFR1OP2-FGFR1 (8-11). However, the effects of these agents on TPR-FGFR1 remain to be elucidated. In the present study, their effects on the proliferation of TPR-FGFR1-expressing Baf3 cells were examined. The data showed that the treatment of TPR-FGFR1-expressing Baf3 cells with TKI258, ponatinib and AZD4547 significantly inhibited cell growth, with $\mathrm{IC}_{50}$ values of $112.374,13.506$ and $8.503 \mathrm{nmol} / 1$, respectively. TKI258 treatment did not affect cell proliferation at the concentration of $10 \mathrm{nmol} / \mathrm{l}$. By contrast, incubation with $10 \mathrm{nmol} / 1$ ponatinib resulted in significant cell growth inhibition $(\mathrm{P}<0.01)$, and treatment with AZD4547 at the same concentration further inhibited the proliferation $(\mathrm{P}<0.01)$. The addition of IL3 resulted in $\mathrm{IC}_{50}$ values of $>500 \mathrm{nmol} / \mathrm{l}$ (Fig. 4), suggesting that the effect of the three drugs were specifically mediated by FGFR1 signaling (9).

TKI258, ponatinib, and AZD4547 induce the apoptosis of TPR-FGFR1-expressing Baf3 cells. To determine the effects of TKI258, ponatinib and AZD4547 on the apoptosis of cells, Annexin V/PI staining was used. According to the effects of the TKIs on cell proliferation, the cells were treated with TKI258 at concentrations of 100 and $1,000 \mathrm{nmol} / \mathrm{l}$, and with ponatinib and AZD4547 at concentrations of 10 and $100 \mathrm{nmol} / \mathrm{l}$. As shown in Fig. 5A and B, exposure to TKI258, ponatinib and AZD4547 at a concentration of $100 \mathrm{nmol} / 1$ increased the apoptotic rate (Annexin $\mathrm{V}^{+} / \mathrm{PI}^{-}$and Annexin ${ }^{+} / \mathrm{PI}^{+}$) from $8.28 \%$ in the control group to $60.90,70.80$ and $53.95 \%$ in the TPR-FGFR1-expressing Baf3 cells, respectively. No significant difference was found among the three TKIs on their effects on cell apoptosis $(\mathrm{P}>0.05)$. Exposure to TKI258 at a concentration of $1,000 \mathrm{nmol} / 1$ markedly increased the apoptotic rate to $96.81 \%$.

TKI258, ponatinib and AZD4547 inhibit the phosphorylation of TPR-FGFR1 and downstream signaling molecules. The phosphorylation of FGFR1 leads to the activation of four key downstream signaling pathways: RAS-RAF-mitogen-activated protein kinase, phosphoinositide 3-kinase-Akt, STAT and PLC $\gamma$ (12). In the present study, western blot analysis was used to assess the effects of TKI258, ponatinib and AZD4547 on the phosphorylation of FGFR1, and the downstream signaling molecules, ERK1/2, Akt, STAT5 and PLC $\gamma$. The data (Fig. 6) showed that $100 \mathrm{nmol} / \mathrm{l}$ ponatinib treatment resulted in a marked reduction in the phosphorylation of ERK1/2, STAT5 and PLC $\gamma$, whereas the same response required $1,000 \mathrm{nmol} / 1$ of TKI258. The effects of AZD4547 on the activation of ERK1/2 and PLC $\gamma$ were similar to the effects of ponatimib, however, it had a more marked effect on the activation of STAT5. Treatment with $10 \mathrm{nmol} / 1$ AZD4547 almost completely inhibited the phosphorylation, whereas a 10 -fold higher concentration of ponatinib was required. The phosphorylation of Akt was not affected by any of the TKIs.

\section{Discussion}

The TPR-FGFRl fusion transcript was first identified in our previous study in 2012 (2). To date, a total of four cases with the $\mathrm{t}(1 ; 8)(\mathrm{q} 25 ; \mathrm{p} 11.2)$ translocation have been reported $(2,5,13,14)$. All the patients showed myeloproliferative neoplasm bone marrow morphology and peripheral monocytosis. Mammalian TPR is a $267-\mathrm{kDa}$ protein, which attaches to the nuclear pore complex (NPC) (15). It contains a large coiled-coil forming amino-terminus, which involves the TprMet and NPC association domain, and an acidic carboxyl-terminus with total protein 
$\mathbf{A}$

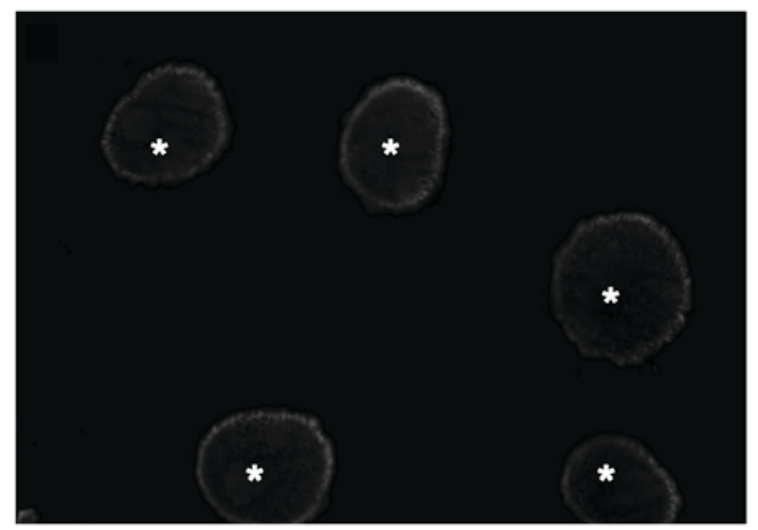

B

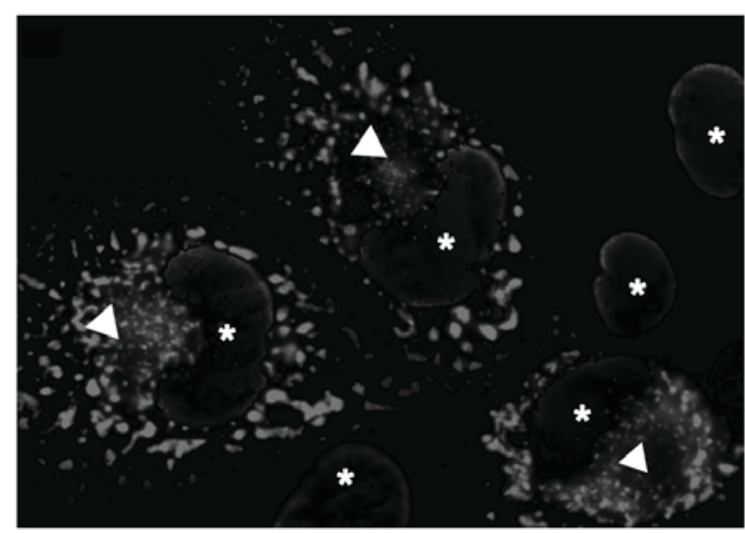

Figure 1. TPR-FGFR1 is localized in the cytoplasm. (A) Untransfected Baf3 cells served as a negative control. (B) COS-1 cells were transiently transfected with the TPR-FGFR1/pEZ-M03 vector. Images were captured with a x100 oil immersion lens. Subcellular localization of TPR-FGFR1 is indicated by GFP $(\Delta)$. DAPI staining was used to detect nuclei $(*)$. TPR, translocated promoter region; FGFR1, fibroblast growth factor receptor 1.

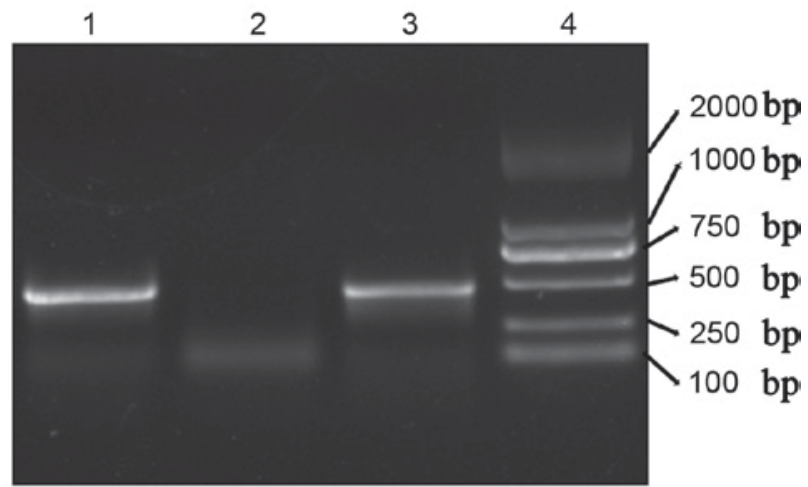

1. TPR-FGFR1-expressing Baf3 cells

2. pMSCV-neo tranfected Baf3 cells

3. TPR-FGFR1/pMSCV construct

4. DL2000

Figure 2. TPR-FGFR1 is expressed in the transfected cells. The mRNA expression of TPR-FGFR1 was detected in the TRP-FGFR1/pMSCV- or empty vector-transfected Baf3 cells using reverse transcription PCR. The PCR product of the TPR-FGFR1/pMSCV construct was used as a positive control. PCR, polymerase chain reaction. TPR, translocated promoter region; FGFR1, fibroblast growth factor receptor 1.

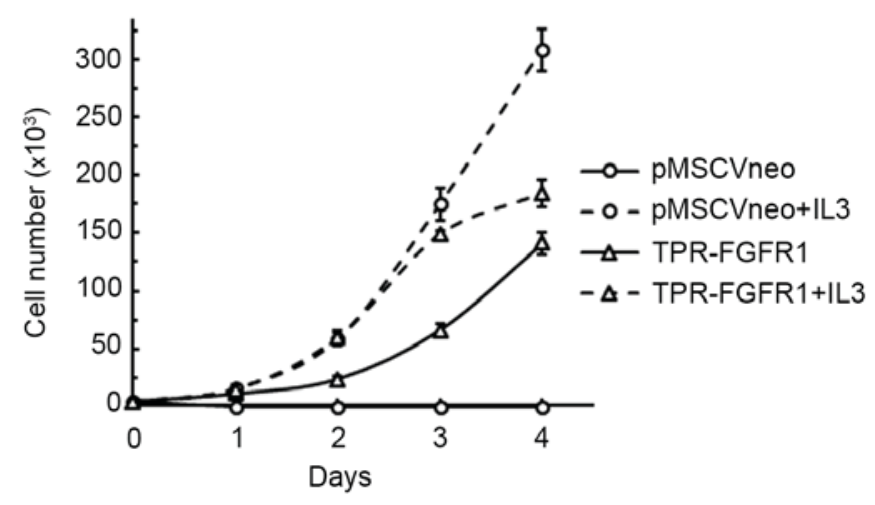

Figure 3. TPR-FGFR1 transforms Baf3 cells into IL-3-independent cells. The TPR-FGFR1- or empty vector-transfected Baf3 cells were cultured with or without IL-3 for 4 days. The number of live cells in each group was counted every day. Data are expressed as the mean \pm standard error of the mean of three independent experiments. TPR, translocated promoter region; FGFR1, fibroblast growth factor receptor 1; IL3, interleukin 3.

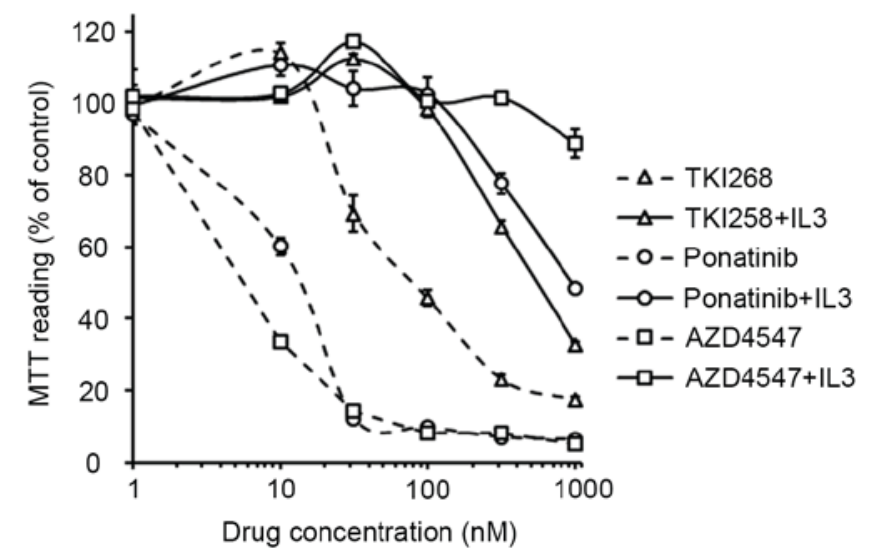

Figure 4. Tyrosine kinase inhibitorsinhibit the proliferation of TPR-FGFR1-expressing Baf3 cells. The TRP-FGFR1-expressing Baf3 cells were treated with increasing concentrations of TKI258, ponatinib or AZD4547 for $48 \mathrm{~h}$. The absorbance of the control group was taken as $100 \%$. Data are of at least three independent experiments. TPR, translocated promoter region; FGFR1, fibroblast growth factor receptor 1; MTT, methylthiazolyldiphenyl-tetrazolium bromide; IL3, interleukin 3 .

kinase and nuclear targeting activity $(16,17)$. In the present study, the TPR-FGFRl fusion gene was stably transfected into hematopoietic Baf3 cells. The resulting data showed that the fusion gene transformed Baf3 cells into IL3-independent cells, which was similar to other EMS fusion genes. The present study also investigated the subcellular localization of TPR-FGFR1, as Bangs et al (17) suggested that the subcellular localization may affect the activity of the fusion protein. The data from the present study showed that TPR-FGFR1 was localized exclusively in the cytoplasm, rather than attaching to the NPC. This was consistent with a previous study, in which the NPC-associated domain and the nuclear targeting motif were necessary for NPC attachment (16), whereas the latter motif was not involved in TPR-FGFR.

Small molecule inhibitors targeting tyrosine kinase activity have been developed for the treatment of various malignancies. Particular success follows the use of imatinib for the treatment of Philadelphia chromosome-positive chronic myeloid leukemia ( $\left.\mathrm{Ph}^{+} \mathrm{CML}\right)(18)$. As EMS is caused by the 

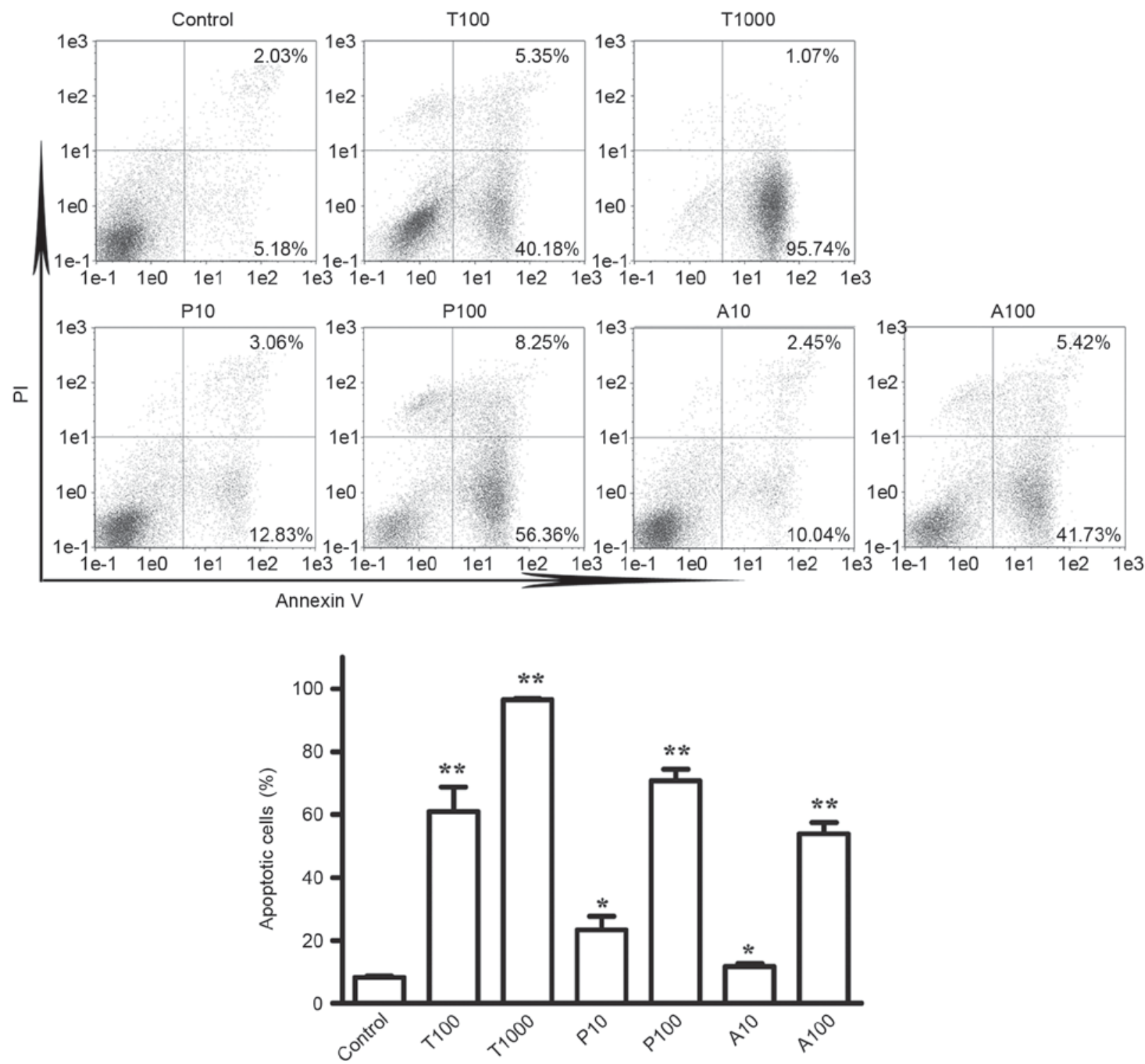

Figure 5. Tyrosine kinase inhibitors induce the apoptosis of TPR-FGFR1-expressing Baf3 cells. (A) Cells were treated with different concentrations of TKI258, ponatinib, or AZD4547 for $24 \mathrm{~h}$. The apoptosis was detected using an Annexin V/PI staining kit. The apoptotic cells were defined as Annexin $\mathrm{V}^{+} / \mathrm{PI}{ }^{-}\left(\mathrm{lower}^{-}\right.$ right quadrant) and Annexin $\mathrm{V}^{+} / \mathrm{PI}^{+}$(upper right quadrant). (B) Quantitative data for the percentages of apoptotic cells were pooled from three independent experiments. ${ }^{*} \mathrm{P}<0.05$, vs. control group. ${ }^{* *} \mathrm{P}<0.01$ vs. control group. TPR, translocated promoter region; FGFR1, fibroblast growth factor receptor 1; T, TK1258; P, ponatinib; A, AZD4547.

constitutive activation of FGFR1, TKIs targeting FGFR1 are promising drugs for the disease. TKI258, ponatinib and AZD4547 are three clinically investigated FGFR inhibitors. Their effects have been evaluated in patients with renal cell carcinoma, breast cancer, relapsed multiple myeloma, urothelial cancer and chronic myelogenous leukemia (19). However, none of these TKIs have been investigated in patients with EMS. As TKI258, ponatinib and AZD4547 are also efficient inhibitors against FGFR1, they may be potential drugs for EMS. This has been partially demonstrated in previous studies, which showed that TKI258, ponatinib and AZD4547 inhibit the growth of ZNF198-FGFR1-, BCR-FGFR1- and CUX-FGFR1-transformed Baf3 cells or FGFR1OP2-FGFR1-positive KG1a cells (8-11). However, the effects of these three TKIs have not been evaluated in the same fusion gene systematically. In the present study, the activities of TKI258, ponatinib and AZD4547 for EMS treatment were compared using TPR-FGFR1-expressing Baf3 cells. The data showed that, although all three TKIs markedly inhibited the proliferation of TPR-FGFR1-expressing Baf3 cells, AZD4547 was the most efficient drug, whereas TKI258 was the least efficient. The $\mathrm{IC}_{50}$ value of TKI258 was $112.374 \mathrm{nmol} / \mathrm{l}$, which was $~ 10$-fold higher, compared with those of ponatinib and AZD4547. In addition, at the concentration of $10 \mathrm{nmol} / \mathrm{l}$, the rate of inhibition following AZD4547 treatment was significantly higher, compared with that of the other TKIs.

In order to confirm the effects of the three TKIs on TPR-FGFR1, western blot analysis was used to determine their activity against FGFR1 signaling. Similar results were obtained. TKI258 was the least efficient drug for the activation of FGFR1 and its downstream signaling molecules, ERK1/2, PLCg, and STAT5, as at least 10-fold higher concentration than ponatinib and AZD4547 was required to achieve the similar effect. Although the effects of AZD4547 and ponatinib 


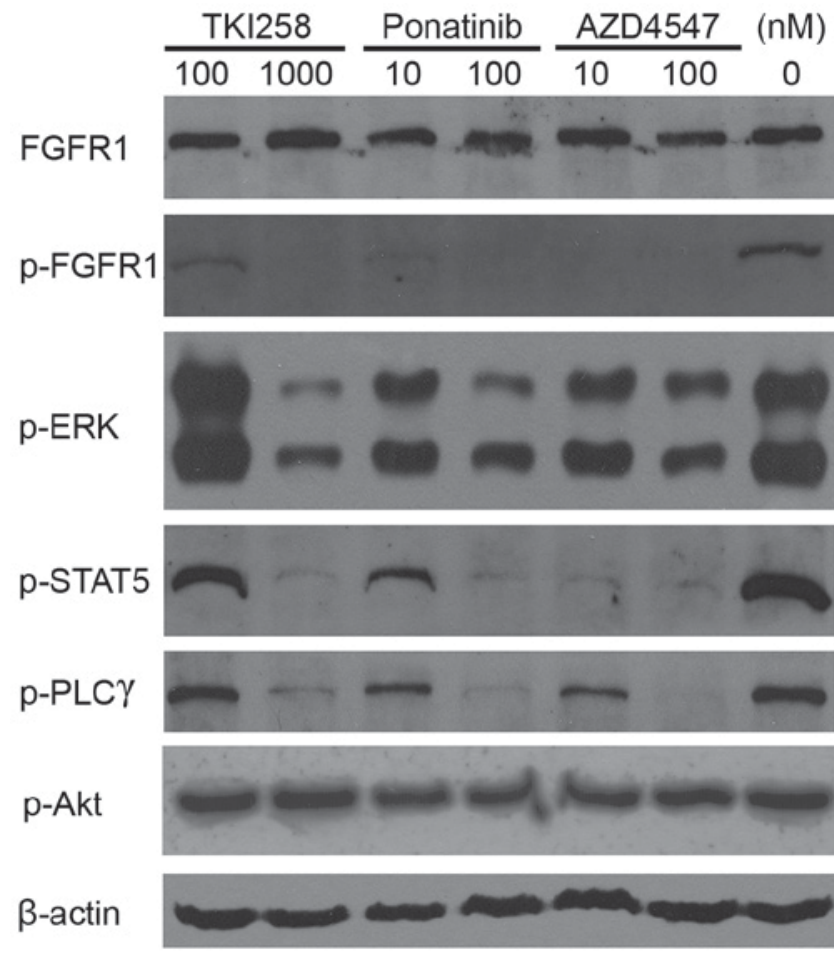

Figure 6. Tytosine kinase inhibitors inhibit FGFR1 activation. The TPR-FGFR1-expressing Baf3 cells were starved overnight and treated with different concentrations of TKI258, ponatinib or AZD4547 for 90 min. The phosphorylation of FGFR1, and its downstream molecules, ERK, STAT5, PLC $\gamma$ and Akt were detected using western blot analysis. $\beta$-actin was used as a loading control. TPR, translocated promoter region; FGFR1, fibroblast growth factor receptor 1; ERK, extracellular signal-regulated kinase; STAT5, signal transducer and activator of transcription; PLC $\gamma$, phospholipase $\gamma$; p-, phosphorylated.

were similar on the activation of ERK1/2 and PLC $\gamma$, AZD4547 was more potent in activating the phosphorylation of STAT5. The treatment of cells with $10 \mathrm{nmol} / \mathrm{l}$ AZD4547 but minimal ponatinib resulted in almost total inhibition of the activation of STAT5. This was consistent with the proliferation data, which showed that the inhibitory rate of $10 \mathrm{nmol} / 1 \mathrm{AZD} 4547$ was significantly higher, compared with that of ponatinib at the same concentration ( 66.5 vs. $39.7 \%$, respectively; $\mathrm{P}<0.01$ ). Taken together, the results suggested that AZD4547 was the most efficient drug for FGFR1 signaling, and the activation of STAT5 was a critical event for TPR-FGFR1-mediated cell transformation.

Ponatinib is a non-selective TKI, which targets breakpoint cluster region (BCR)-Abelson (ABL), FGFR, vascular endothelial growth factor receptor (VEGFR) and platelet-derived growth factor receptor, with similar enzyme $\mathrm{IC}_{50}$ values. It is now approved for the treatment of CML or patients with $\mathrm{Ph}^{+}$acute lymphocytic leukemia with $\mathrm{T}^{3151}$ mutations, or those for whom all other TKIs have failed. The indication of the drug has been narrowed due to severe cardiovascular events; however, the mechanisms underlying these adverse events remain to be fully elucidated. They may be induced by BCR-ABL-specific effects, as cardiovascular adverse events have also been identified in patients treated with other BCR-ABL-targeted TKIs (20). However, further investigations are required to verify this possibility, as the majority of trials of ponatinib focus on CML therapy, whereas adverse events in other malignancies remain to be fully elucidated. The activity of ponatinib against VEGFR may be another explanation for the adverse events. Previous studies have indicated that VEGFR inhibitors increase the risk of pancreatitis and treatment-associated mortality rates $(21,22)$. As a result, compared with ponatinib and TKI258, AZD4547 may be safer for clinical use, as the specificity of AZD4547 eliminates the adverse events associated with VEGFR.

A previous in vivo study showed that, despite ponatinib inhibiting the proliferation of KGla cells with an $\mathrm{IC}_{50}$ value of $20 \mathrm{nmol} / 1$, treatment with $30 \mathrm{mg} / \mathrm{kg}$ ponatinib, which resulted in a mean plasma level of $561 \mathrm{nmol} / \mathrm{l}$ following $6 \mathrm{~h}$ of drug administration, only prolonged the survival rates of $40 \%$ of mice with xenograft KG1 cells (23). This suggests that treatment with a single TKI may not be sufficient for EMS treatment. The data obtained in the present study indicated that the activation of another FGFR downstream signaling molecule, Akt, was not affected by the three TKIs. Akt signaling remains active in hematological malignancies, and is further activated by ZNF198-FGFR1 and FOP-FGFR1 transformation (11,24-26). Clinical trials have indicated that the combined therapy of Akt inhibitors with bortezomib or lenalidomide significantly improves the survival rates and responses of patients with multiple myeloma $(27,28)$. Therefore, combining TKIs and Akt inhibitors may be a novel strategy for the treatment of EMS.

In conclusion, the data obtained in the present study indicated that AZD4547 showed increased potency, compared with TKI258 and ponatinib, on the inhibition of cell proliferation and FGFR1 signaling. Considering the adverse events associated with VEGFR inhibitors, AZD4547 may be the most promising drug for the treatment of EMS.

\section{References}

1. Jackson CC, Medeiros LJ and Miranda RN: 8p11 myeloproliferative syndrome: A review. Hum Pathol 41: 461-476, 2010.

2. Li F, Zhai YP, Tang YM, Wang LP and Wan PJ: Identification of a novel partner gene, TPR, fused to FGFR1 in 8p11 myeloproliferative syndrome. Genes Chromosomes Cancer 51: 890-897, 2012.

3. Gervais C, Dano L, Perrusson N, Hélias C, Jeandidier E, Galoisy AC, Ittel A, Herbrecht R, Bilger K and Mauvieux L: A translocation $\mathrm{t}(2 ; 8)(\mathrm{q} 12 ; \mathrm{p} 11)$ fuses FGFR1 to a novel partner gene, RANBP2/NUP358, in a myeloproliferative/myelodysplastic neoplasm. Leukemia 27: 1186-1188, 2013.

4. Nakamura Y, Ito Y, Wakimoto N, Kakegawa E, Uchida Y and Bessho M: A novel fusion of SQSTM1 and FGFR1 in a patient with acute myelomonocytic leukemia with $\mathrm{t}(5 ; 8)(\mathrm{q} 35 ; \mathrm{p} 11)$ translocation. Blood Cancer J 4: e265, 2014.

5. Kim SY, Kim JE, Park S and Kim HK: Molecular identification of a TPR-FGFR1 fusion transcript in an adult with myeloproliferative neoplasm, T-lymphoblastic lymphoma, and a $\mathrm{t}(1 ; 8)$ (q25;p11.2). Cancer Genet 207: 258-262, 2014.

6. Grand EK, Chase AJ, Heath C, Rahemtulla A and Cross NC: Targeting FGFR3 in multiple myeloma: Inhibition of $\mathrm{t}(4 ; 14)$-positive cells by SU5402 and PD173074. Leukemia 18: 962-966, 2004.

7. Chen J, Deangelo DJ, Kutok JL, Williams IR, Lee BH, Wadleigh M, Duclos N, Cohen S, Adelsperger J, Okabe R, et al: PKC412 inhibits the zinc finger 198-fibroblast growth factor receptor 1 fusion tyrosine kinase and is active in treatment of stem cell myeloproliferative disorder. Proc Natl Acad Sci USA 101: 14479-14484, 2004.

8. Chase A, Grand FH and Cross NC: Activity of TKI258 against primary cells and cell lines with FGFR1 fusion genes associated with the $8 \mathrm{p} 11$ myeloproliferative syndrome. Blood 110: 3729-3734, 2007. 
9. Wasag B, Lierman E, Meeus P, Cools J and Vandenberghe P The kinase inhibitor TKI258 is active against the novel CUX1-FGFR1 fusion detected in a patient with T-lymphoblastic leukemia/lymphoma and t(7;8)(q22;p11). Haematologica 96 : 922-926, 2011

10. Chase A, Bryant C, Score J and Cross NC: Ponatinib as targeted therapy for FGFR 1 fusions associated with the $8 \mathrm{p} 11$ myeloproliferative syndrome. Haematologica 98: 103-106, 2013.

11. Gavine PR, Mooney L, Kilgour E, Thomas AP, Al-Kadhimi K, Beck S, Rooney C, Coleman T, Baker D, Mellor MJ, et al AZD4547: An orally bioavailable, potent, and selective inhibitor of the fibroblast growth factor receptor tyrosine kinase family. Cancer Res 72: 2045-2056, 2012.

12. Turner $\mathrm{N}$ and Grose R: Fibroblast growth factor signalling: From development to cancer. Nat Rev Cancer 10: 116-129, 2010.

13. Yoshida C, Takeuchi M and Sadahira Y: A novel t(1;8)(q25;p11.2) translocation associated with $8 \mathrm{p} 11$ myeloproliferative syndrome. Br J Haematol 156: 271-273, 2012.

14. Kim WS, Park SG, Park G, Jang SJ, Moon DS and Kang SH: 8p11 myeloproliferative syndrome with $\mathrm{t}(1 ; 8)(\mathrm{q} 25 ; \mathrm{p} 11.2)$ : A case report and review of the literature. Acta Haematol 133: 101-105, 2015.

15. Hase ME, Kuznetsov NV and Cordes VC: Amino acid substitutions of coiled-coil protein Tpr abrogate anchorage to the nuclear pore complex but not parallel, in-register homodimerization. Mol Biol Cell 12: 2433-2452, 2001.

16. Cordes VC, Hase ME and Muller L: Molecular segments of protein Tpr that confer nuclear targeting and association with the nuclear pore complex. Exp Cell Res 245: 43-56, 1998.

17. Bangs P, Burke B, Powers C, Craig R, Purohit A and Doxsey S: Functional analysis of Tpr: Identification of nuclear pore complex association and nuclear localization domains and a role in mRNA export. J Cell Biol 143: 1801-1812, 1998.

18. Giles FJ, Mauro MJ, Hong F, Ortmann CE, McNeill C, Woodman RC, Hochhaus A, le Coutre PD and Saglio G: Rates of peripheral arterial occlusive disease in patients with chronic myeloid leukemia in the chronic phase treated with imatinib, nilotinib, or non-tyrosine kinase therapy: A retrospective cohort analysis. Leukemia 27: 1310-1315, 2013.

19. Katoh $M$ and Nakagama H: FGF receptors: Cancer biology and therapeutics. Med Res Rev 34: 280-300, 2014.

20. Loren CP, Aslan JE, Rigg RA, Nowak MS, Healy LD, Gruber A, Druker BJ and McCarty OJ: The BCR-ABL inhibitor ponatinib inhibits platelet immunoreceptor tyrosine-based activation motif (ITAM) signaling, platelet activation and aggregate formation under shear. Thromb Res 135: 155-160, 2015.
21. Ghatalia P, Morgan CJ, Choueiri TK, Rocha P, Naik G and Sonpavde G: Pancreatitis with vascular endothelial growth factor receptor tyrosine kinase inhibitors. Crit Rev Oncol Hematol 94: 136-145, 2014

22. Schutz FA, Je Y, Richards CJ and Choueiri TK: Meta-analysis of randomized controlled trials for the incidence and risk of treatment-related mortality in patients with cancer treated with vascular endothelial growth factor tyrosine kinase inhibitors. J Clin Oncol 30: 871-877, 2012.

23. Ren M, Qin H, Ren R and Cowell JK: Ponatinib suppresses the development of myeloid and lymphoid malignancies associated with FGFR1 abnormalities. Leukemia 27: 32-40, 2013.

24. Dong S, Kang S, Gu TL, Kardar S, Fu H, Lonial S, Khoury HJ, Khuri $\mathrm{F}$ and Chen J: 14-3-3 integrates prosurvival signals mediated by the AKT and MAPK pathways in ZNF198-FGFR1-transformed hematopoietic cells. Blood 110: 360-369, 2007.

25. Guasch G, Ollendorff V, Borg JP, Birnbaum D and Pébusque MJ: 8p12 stem cell myeloproliferative disorder: The FOP-fibroblast growth factor receptor 1 fusion protein of the $t(6 ; 8)$ translocation induces cell survival mediated by mitogen-activated protein kinase and phosphatidylinositol 3-kinase/Akt/mTOR pathways. Mol Cell Biol 21: 8129-8142, 2001.

26. Hideshima T, Catley L, Yasui H, Ishitsuka K, Raje N, Mitsiades C, Podar K, Munshi NC, Chauhan D, Richardson PG and Anderson KC: Perifosine, an oral bioactive novel alkylphospholipid, inhibits Akt and induces in vitro and in vivo cytotoxicity in human multiple myeloma cells. Blood 107: 4053-4062, 2006.

27. Richardson PG, Wolf J, Jakubowiak A, Zonder J, Lonial S, Irwin D, Densmore J, Krishnan A, Raje N, Bar M, et al: Perifosine plus bortezomib and dexamethasone in patients with relapsed/refractory multiple myeloma previously treated with bortezomib: Results of a multicenter phase I/II trial. J Clin Oncol 29: 4243-4249, 2011.

28. Jakubowiak AJ, Richardson PG, Zimmerman T, Alsina M, Kaufman JL, Kandarpa M, Kraftson S, Ross CW, Harvey C, Hideshima T, et al: Perifosine plus lenalidomide and dexamethasone in relapsed and relapsed/refractory multiple myeloma: A phase I multiple myeloma research consortium study. Br J Haematol 158: 472-480, 2012. 